\title{
CORRESPONDENCE
}

\section{Tongue as the Window to the Heart}

\author{
Tsung O. Cheng
}

\section{To the Editor:}

I read with great interest your masterful article on study of the tongue in patients with coronary heart disease. ${ }^{(1)}$ Tongue is, indeed, the window to the heart. ${ }^{(2)}$ Many cardiovascular diseases including coronary heart disease can be accurately diagnosed by the Chinese traditional physicians by careful inspection of the tongue (Figure 1).

\section{REFERENCES}

1. Feng $Y, X u H, Q u D$, Zheng F, Shi DZ, Chen KJ. Study on the tongue manifestations for the blood-stasis and toxin syndrome in the stable patients of coronary heart disease. Chin J Integr Med 2011;17:333-338.

2. Cheng TO: The evolution of cardiology in China. In: Wan S, Yim APC, eds. Cardiothoracic surgery in China: past, present and future. Hong Kong: The Chinese University Press; 2007:204-305.
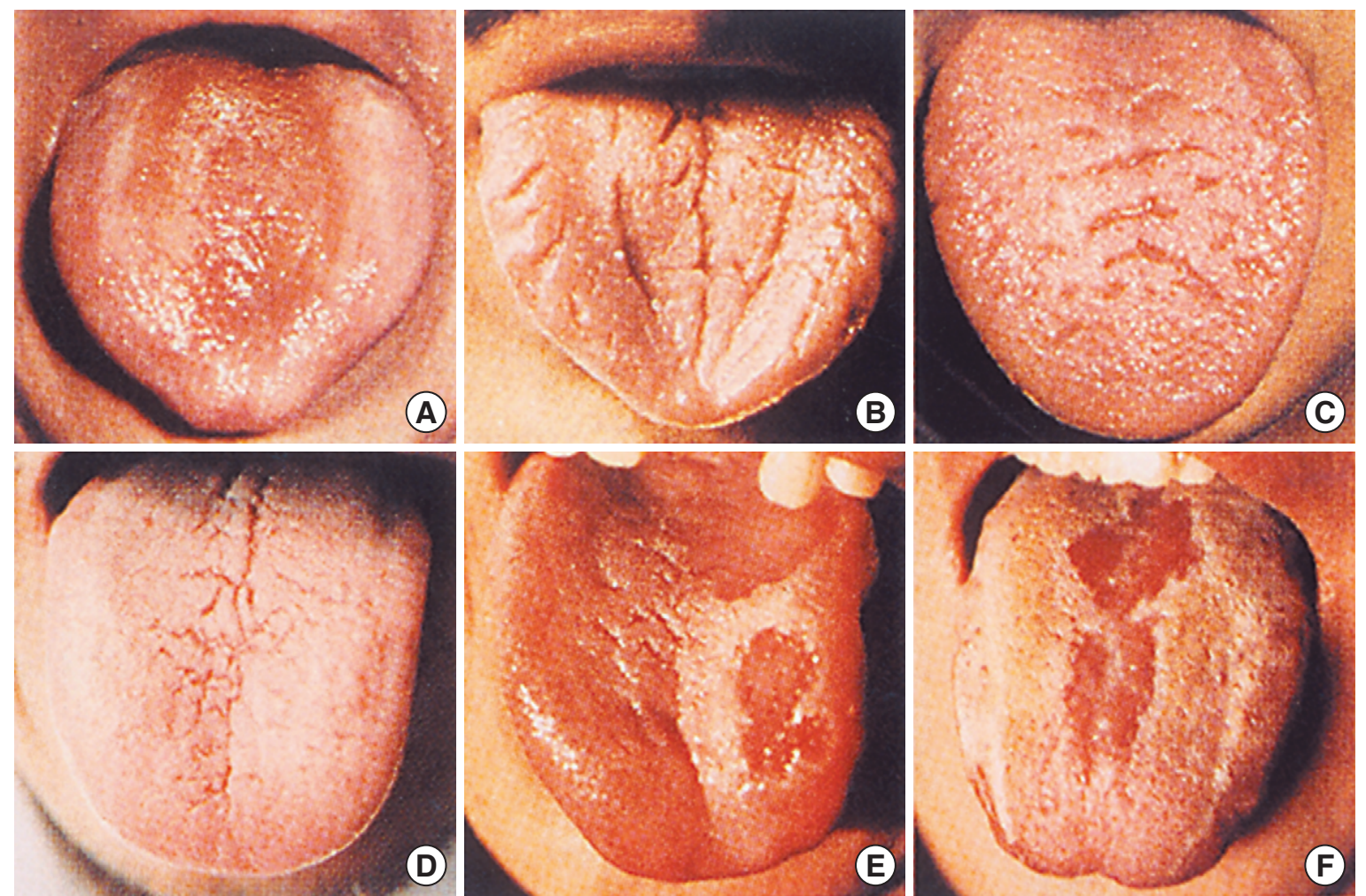

Figure 1. Tongue Appearances in Various Cardiovascular Diseases

Notes: A: normal coated tongue of a cigarette smoker with nicotine staining; B: normal fissured tongue ("scrotal tongue"); C: transverse fissuring of tongue in Down syndrome; D: combined horizontal, vertical, and diagonal fissuring of the tongue in a patient with cardiac arrhythmias; E: geographical tongue in a patient with coronary artery disease and cerebral artery spasm; F: chicken heart tongue in a patient with coronary artery disease

(Received June 2, 2011) Edited by YUAN Lin

CThe Chinese Journal of Integrated Traditional and Western Medicine Press and Springer-Verlag Berlin Heidelberg 2011 The George Washington University Medical Centre, Washington, D.C. 20037 , U.S.A.

Tel: 202-741-2426, E-mail: tcheng @mfa.gwu.edu DOI: $10.1007 / \mathrm{s} 11655-011-0824-x$ 